OMS define el carcinoma sarcomatoide como tumor maligno con evidencia morfológica y/o inmunohistoquímica de diferenciación epitelial y mesenquimal, especificando en el diagnóstico la presencia o ausencia de elementos heterólogos ${ }^{9}$. Algunos autores consideran que el carcinosarcoma y el carcinoma sarcomatoide son entidades diferentes, reservando el primer término para los casos en que existían elementos mesenquimales heterólogos. Otros argumentan que los detalles histológicos diferenciales no tienen significado clínico por lo que agrupan todos estos tumores en una misma entidad llamada "carcinoma sarcomatoide». Esta es la tendencia actual que se refleja en la clasificación $\mathrm{OMS}^{10}$. La cistectomía radical, cuando es posible, parece ser el tratamiento de elección, aunque muchos pacientes desarrollan posteriormente recidivas locales o metástasis a distancia. La radioterapia y quimioterapia adyuvante se han utilizado con distinto éxito ${ }^{4}$. En cuanto al pronóstico, es infausto en la mayoría de las ocasiones, con una supervivencia a los 5 años del 20\%. El factor pronóstico más influyente es el estadio ${ }^{2}$. En definitiva, el carcinosarcoma es un tumor agresivo, de histología compleja y mal pronóstico. El escaso número de casos publicados hace difícil establecer un tratamiento estandarizado del mismo.

B I B L I O G R A F Í A

1. Arguelles Salido E, Travado Soria P, Perez Espejo MP, Rodriguez Corchero J, Medina Lopez RA, Pena Outeirino JM. Carcinosarcoma vesical: Análisis de nuestra serie y revisión de la literatura. Actas Urol Esp. 2004;28:262-8.

2. Wick MR, Swanson PE. Carcinosarcomas: current perspectives and an historical review of nosological concepts. Semin Diagn Pathol. 1993;10:118-27.

3. Lopez-Beltran A, Pacelli A, Rothenberg HJ, Wollan PC, Zincke H, Blute ML, et al. Carcinosarcoma and sarcomatoid carcinoma of the bladder: clinicopathological study of 41 cases. J Urol. 1998; 159:1497-503.
4. Baschinsky DY, Chen JH, Vadmal MS, Lucas JG, Bahnson RR, Niemann TH. Carcinosarcoma of the urinary bladder-an aggressive tumor with diverse histogenesis. A clinicopathologic study of 4 cases and review of the literature. Arch Pathol Lab Med. 2000;124:1172-8.

5. Perret L, Chaubert P, Hessler D, Guillou L. Primary heterologous carcinosarcoma (metaplastic carcinoma) of the urinary bladder: a clinicopathologic, immunohistochemical, and ultrastructural analysis of eight cases and a review of the literature. Cancer. 1998;82:1535-49.

6. Li Y, Outman JE, Mathur SC. Carcinosarcoma with a large cell neuroendocrine epithelial component: first report of an unusual biphasic tumour of the urinary bladder. J Clin Pathol. 2004;57:318-20.

7. Torenbeek R, Blomjous CE, de Bruin PC, Newling DW, Meijer CJ. Sarcomatoid carcinoma of the urinary bladder. Clinicopathologic analysis of 18 cases with immunohistochemical and electron microscopic findings. Am J Surg Pathol. 1994;18: 241-9.

8. Thompson L, Chang B, Barsky SH. Monoclonal origins of malignant mixed tumors (carcinosarcomas). Evidence for a divergent histogenesis. Am J Surg Pathol. 1996;20:277-85.

9. López Beltrán A, Sauter G, Gasser T, Hartmann A, SchmitzDräger H. Infiltranting urothelial carcinoma; WHO classification of noninvasive papillary urothelial tumors. World Health Organization classification of tumors. En: Eble JN, Epstein JI, Sesterhenn I, editors. Pathology and genetics of tumors of the urinary system and male genital organs. Lyon: IARCC; 2004.

10. Picazo ML, Regojo RM, Gonzalez-Peramato P. Variantes histológicas del carcinoma urotelial con implicaciones diagnósticas, pronósticas y terapéuticas. Actas Urol Esp. 2007;31: 989-1001.

M. Alvarez*, V. Hernández, N. Amaruch y C. Llorente

Servicio de Urología, Hospital Universitario Fundación Alcorcón, Madrid, España

*Autor para correspondencia.

Correos electrónicos: gonzag1@hotmail.com, malvareza@fhalcorcon.es (M. Alvarez).

\title{
Liposarcoma desdiferenciado de alto grado de cordón espermático. A propósito de un caso
}

\section{High grade dedifferenciated liposarcoma. Case report and review of the literature}

\section{Sr. Director:}

Las neoplasias paratesticulares derivan del cordón espermático y de los anexos testiculares, y suelen ser bien diferenciadas. En un tercio de los casos pueden presentarse tumores malignos, siendo los más frecuentes los sarcomas (encontrando como variedad dentro de ellos el liposarcoma).

Presentamos el caso de un paciente de 61 años sin antecedentes de interés que consultó por una masa inguinoescrotal indolora de 3 años de evolución, indurada, que no transiluminaba, independiente del testículo. No se palparon adenopatías. Se realizó una TC abdómino pélvica, que evidenció una tumoración de $5 \mathrm{~cm}$ dependiente del cordón espermático, sólida, con captación de contraste, bien delimitada. No se visualizaron adenopatías inguinales o pélvicas de tamaño significativo. El estudio hepático y pulmonar no demostró alteraciones. Los marcadores tumorales fueron: antígeno carcinoembrionario $0,8 \mathrm{ng} / \mathrm{ml}$, 


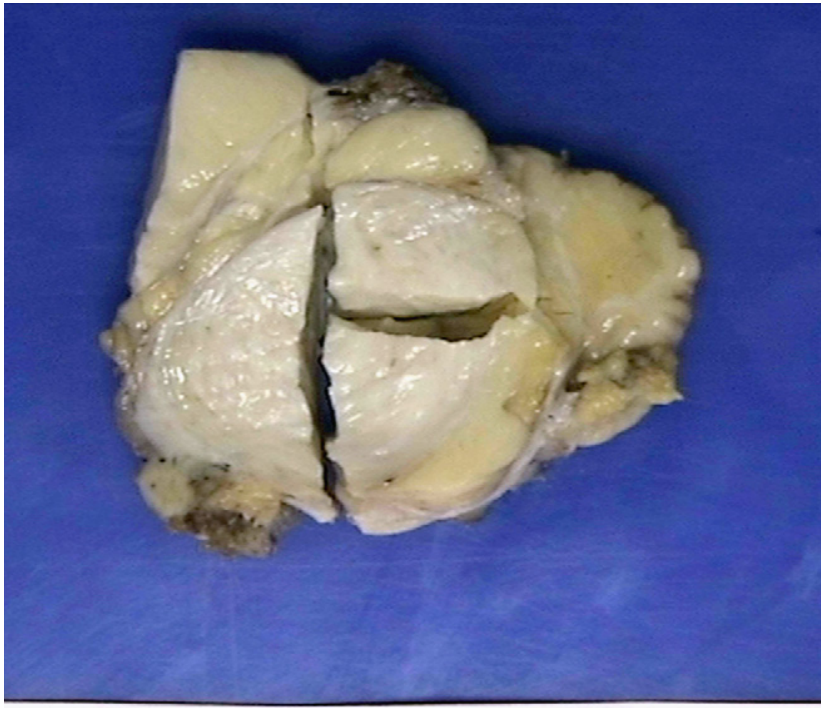

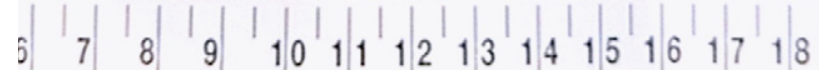

Figura 1 - Masa paratesticular extirpada; aspecto macroscópico.

alfafetroproteina $2,6 \mathrm{ng} / \mathrm{ml}$, PSA $0,79 \mathrm{ng} / \mathrm{ml}$. Se realizó una orquiectomía radical, incluyendo dicha masa, por vía inguinal.

El examen anatomopatológico (fig. 1) mostró una masa blancoamarillenta multilobulada, encapsulada y bien delimitada de $9 \times 7 \times 7 \mathrm{~cm}$. En el estudio microscópico (fig. 2) se describió, una lesión mesenquimal maligna con múltiples patrones de diferenciación, conteniendo áreas lipomatosas con lipoblastos y con un índice proliferativo de Ki67 aumentado. Había áreas de aspecto fibrosarcomatoso, mixofibrosarcomatoso, neural y leiomiosarcomatoso. Mostró positividad para actina, desmina, vimentina y S-100. Los límites quirúrgicos eran negativos. Todo ello completó el diagnóstico de liposarcoma desdiferenciado de alto grado de cordón espermático. A los 21 meses de la intervención el paciente se encuentra asintomático y sin recidiva de la enfermedad local o a distancia.

Los tumores paratesticulares son las neoplasias derivadas del cordón espermático más frecuentes. Un tercio son malignos, siendo los más frecuentes los sarcomas ${ }^{1}$.

El liposarcoma es un tumor maligno de partes blandas que suele presentarse en la edad adulta (entre la quinta y la séptima década de la vida), sobre todo en el retroperitoneo. Constituye el 16-18\% de los sarcomas de partes blandas e incluye 5 subtipos histológicos: bien diferenciado, lipoblástico, fibroblástico, mixoide/células redondas y pleomórfico ${ }^{2}$. Al diagnóstico sólo $4 \%$ de los liposarcomas presentan metástasis. El liposarcoma paratesticular representa el 3-7\% de los sarcomas paratesticulares ${ }^{3,4}$. Aunque los liposarcomas paratesticulares suelen ser bien diferenciados, en ocasiones tiene lugar un proceso de desdiferenciación o progresión a un tumor de mayor grado ${ }^{2,5}$.

Los liposarcomas desdiferenciados se definen como tumores primarios o recurrentes en los que existe un liposarcoma bien diferenciado que asocia o progresa a un sarcoma de alto grado no lipogénico. La presencia de células atípicas, núcleos hipercromáticos, lipoblastos y septos fibrosos nos permiten diferenciarlos de los lipomas. Este tipo de tumores desdiferenciados, a su vez, puede presentar gran variedad de patrones histológicos,

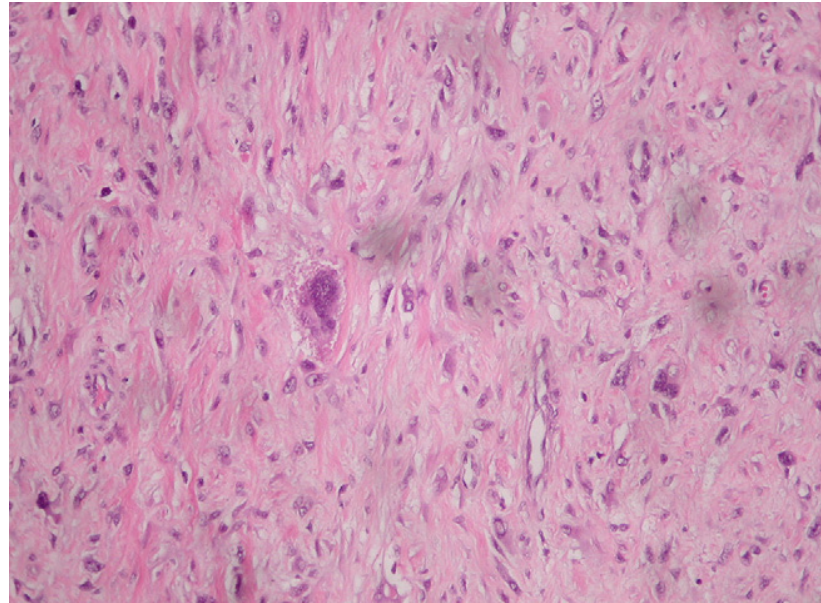

Figura 2 - Tinción de hematoxilina eosina.

siendo el más frecuente el de mixofibrosarcoma de medio-alto grado. Las técnicas inmunohistoquímicas son esenciales para alcanzar el diagnóstico; este tipo de tumores expresa la proteína S-100, desmina, vimentina y proteína CD34. Suelen localizarse en el retroperitoneo, extremidades, cordón espermático y rara vez en la cabeza o cuello ${ }^{2}$.

La forma de presentación de este tipo de tumores es como una masa inguinoescrotal irregular, que no transilumina; en ocasiones se acompañan de un hidrocele reactivo ${ }^{2}$. Se debe plantear el diagnóstico diferencial con la hernia inguinal, el quiste de cordón, el hematocele, el hidrocele, la orquiepididimitis tuberculosa o los tumores testiculares. Ecográficamente se trata de una lesión sólida, hiperecoica y heterogénea. La TC permite la identificación de una lesión hipointensa respecto a la grasa subcutánea, con septos, así como buscar adenopatías pélvicas o retroperitoneales ${ }^{6}$. El estudio de extensión puede completarse con una Rx de tórax y una RM inguinoescrotal.

Dado que se trata de tumores poco frecuentes ${ }^{7}$, los datos acerca del tratamiento y de la supervivencia provienen únicamente de revisiones retrospectivas de grupos puntuales con series poco numerosas de pacientes. Únicamente existe consenso en la importancia que tiene la extirpación de todo el tejido afecto (orquiectomía radical con resección local amplia del tejido blando circundante), siendo uno de los factores pronósticos más importantes la existencia de márgenes quirúrgicos negativos ${ }^{8}$. La linfadenectomía no ha demostrado mejorar la supervivencia ${ }^{4}$.

Los liposarcomas desdiferenciados son de peor pronóstico que los bien diferenciados, aunque menos agresivos que los sarcomas de alto grado. La recurrencia local puede aparecer en el escroto, en el área inguinal o en la pelvis. A falta de estudios adecuados, el papel de la radioterapia o quimioterapia como tratamientos adjuvantes sigue siendo controvertido ${ }^{8}$.

Los márgenes positivos, la localización inguinal, el grado nuclear y de diferenciación guardan relación con el riesgo de recurrencia o progresión ${ }^{8}$. La supervivencia global a 5 años de los liposarcomas es del $70 \%$. El tiempo medio libre de enfermedad es de 36 meses (6 meses-16 años) ${ }^{4}$. Según las series publicadas, a 5 años, se produce una recurrencia local en el $19,4 \%$, metástasis a distancia en el 11,1\% y enfermedad ganglionar pélvica en el $5,5 \%^{8}$. 
1. Coleman J, Brennan MF, Alektiar K, Russo P. Adult spermatic cord sarcomas: management and results. Ann Surg Oncol. 2003;10:669-75.

2. Peyrí E, Urban A, Martínez M, Sanmarti B. Liposarcoma dediferenciado del cordón espermático: degeneración de un lipoma previo. Actas Urol Esp. 2003;27:383-6.

3. Schwartz SL, Swierzewski III SJ, Sondak VK, Grossman HB. Liposarcoma of the spermatic cord: report of 6 cases and review of the literature. J Urol. 1995;153:154-7.

4. Soler J, Zuluaga A, Hidalgo M. Liposarcoma de cordón espermático: aportación de un nuevo caso y revisión de la literatura. Actas Urol Esp. 1999;23:447-54.

5. Henricks WH, Chu YC, Goldblum JR, Weiss SW. Dedifferentiated liposarcoma: a clinicopathological analysis of 155 cases with a proposal for an expanded definition of dedifferentiation. Am J Surg Pathol. 1997;21:271-81.
6. Calahorra F, Pérez C, Ramos A. Liposarcoma paratesticular del cordón espermático. Actas Urol Esp. 1990;3:202-4.

7. Garcia Morua A, Lozano Salinas JF, Valdes Sepulveda F. Liposarcoma of the espermatic cord: our experience and review of the literature. Actas Urol Esp. 2009;33:811-5.

8. Ballo MT, Zagars GK, Pisters PW. Spermatic cord sarcoma: outcome, patterns of failure and management. J Urol. 2001;166: 1306-10.

G. García-Fadrique*, E. Morán Pascual, G. Morales Solchaga, A. Soto, J.F. Morera y J.F. Jiménez-Cruz

Servicio de Urología, Hospital Universitario La Fe, Valencia, España

Autor para correspondencia.

Correo electrónico: gonzag1@hotmail.com

(G. García-Fadrique).

\section{Anuria intermitente postrasplante renal secundaria a granuloma calcificado del neomeato}

\section{Post-renal transplantation intermittent anuria secondary to calcified granuloma of the neomeatus}

\section{Sr. Director:}

Reportamos el caso de una paciente que presenta una obstrucción ureteral temprana secundaria a un granuloma calcificado de la unión ureterovesical después de un trasplante renal heterotópico derecho. No se ha descrito en la literatura esta variante de granuloma.

Se trata de una mujer de 34 años con insuficiencia renal crónica terminal (IRCT) secundaria a lupus eritematoso sistémico diagnosticado en 1990 que ingresa en el programa de hemodiálisis en el año 2000. Presenta antecedentes de hipersensibilización, hiperparatiroidismo secundario a IRC en tratamiento con Cinacalcet (Mimpara) y Calcitriol (Rocaltrol) e HTA en tratamiento con Atenolol, Doxazocina y Amlodipino.

Ingresó en marzo de 2008 para trasplante renal procedente de donante vivo relacionado (esposo). Se realiza, en el donante, nefrectomía izquierda por laparoscopia sin incidencias, obteniéndose un riñón de arteria y vena único y un uréter bien vascularizado y longitud correcta. Como parte del protocolo de desensibilización, la paciente recibió 2 dosis de Rituximab, 6 sesiones de inmunoabsorción pre trasplante así como 3 dosis de Gammaglobulina Monoclonal e inducción con Timoglobulina. Como tratamiento inmunosupresor posquirúrgico, Micofenolato Sódico $750 \mathrm{mg}$ v.o/12 h, Tacrolimus $6 \mathrm{mg}$ v.o/12 h, y Prednisona en esquema de disminución progresiva hasta $20 \mathrm{mg}$ v.o/d.

Se realizó el trasplante heterotópico en fosa renal derecha anastomosando la vena renal término lateral a la vena iliaca externa; la arteria renal a la arteria iliaca externa término lateral utilizando sutura continua de prolene 6 y 7/0 respectivamente con correcta perfusión tras desclampaje y sin complicaciones, realizando una implantación ureterovesical tipo Politano-Leabetter, anclando el extremo distal del uréter con 3 puntos sueltos de poliglecaprone (Monocril) 6/0, con diuresis inmediata. El tiempo de isquemia caliente fue de $2 \mathrm{~min}$ y de isquemia total fue de 50 min. Durante el postoperatorio, se realizó ecografía doppler renal que fue normal y un renograma isotópico con captación adecuada. La paciente presentó mejoría de la función renal con creatinina de $0,8 \mathrm{mg} / \mathrm{dl}$ al alta.

La paciente reingresó a los 14 días por dolor a nivel del injerto, anuria y deterioro de la función renal pasando la creatinina de 0,8 a 2,3 mg/dl. Se solicitó ecografía abdominal que informa de ectasia pieloureteral hasta la anastomosis ureterovesical donde se describe una imagen hiperecogénica compatible con litiasis. Reinicia diuresis de forma espontánea descendiendo los niveles de creatinina a $0,5 \mathrm{mg} / \mathrm{dl}$. Se complementa estudio de la lesión con TC abdominal que informa de ectasia pieloureteral del injerto renal y dilatación ureteral con la presencia de una masa calcificada intravesical en la zona de la anastomosis ureteral del injerto que podría corresponder a un hematoma o un granuloma calcificado (fig. 1). Se practicó citoscopia, evidenciando el neomeato calcificado.

Desde el punto de vista analítico, se objetivó hipercalcemia de 13,1 mg/dl (n: 8,4-10,2 mg/dl) en contexto de un hiperparatiroidismo secundario agravado por la suspensión semanas antes del Cinacalcet (Mimpara), el cual, tras medidas de 